\title{
Miniaturized silicon strain gauge elements to accurately measure mechanical quantities
}

\author{
Th. Frank, M. Khatri, M. Fiedler, A. Gruen, M. Kermann, A. Cyriax, T. Ortlepp, CiS Forschungsinstitut \\ für Mikrosensorik GmbH, Konrad-Zuse-Str. 14, 99099 Erfurt, Deutschland, tfrank@cismst.de
}

\begin{abstract}
Force measurements in microsystems technology are usually based on the piezoresistive effect. Mechanical stress in the doped areas, leads to change of the conduction mechanism, which changes the ohmic resistance. Here, the piezoresistive resistors are monolithically integrated directly into the silicon component by diffusion or implantation. Compared with standard metal strain gauges, the semiconductor integrated resistors have a higher gauge factor and a significantly higher long-term stability. A major advantage of using single-crystal material is the lack of dislocations and grain boundaries. This paper presents some advantageous applications of the developed silicon strain gauge.
\end{abstract}

Key words: silicon strain gauge, wheatstone bridge, stress, semiconductor, piezoresistive coefficient

\section{Introduction}

In order to measure basic mechanical quantities such as pressure, stress, torque, acceleration and mass, it is necessary to measure the applied force. The most commonly used measuring principle is based on the piezoresistive effect. It is used by connecting four resistors to a wheatstone bridge.

In macro applications today, foil-based strain gauges with metallic conductor lines consisting of primarily copper nickel manganese alloys (brand name constantan) are being used [1]. This alloy is characterized by an excellent low temperature coefficient of resistance approximately 1E-5 / K. Compared to pure metals (copper, silver approx $\approx 4 \mathrm{E}-3 / \mathrm{K}$ ) it is lower by a factor of 400 [2]. Many experimental stress and strain analyses are possible due to the simple usage of foil strain gauges. Measurement principles based on the piezoresitive effect are also used in microsystems technology. In contrast to metals, the mechanical stress in doped regions results in a change of the conductivity, which then leads to change of the ohmic resistance. Here, the piezoresistive resistors are monolithically integrated directly into the silicon component by diffusion or implantation. The main application is found in pressure sensors. Compared with the metal strain gauges the semiconductor integrated resistors have a higher gauge factor and a significantly higher long-term stability. A major advantage of using single-crystal material is the lack of dislocations and grain boundaries. In ductile metal, these lead to increased uncertainty as a result of permanent plastic deformation (plastic flow). In the case of monolithic silicon sensors two doping variations are common. In most commonly used method the resistive elements can be realized in the n-type base material by doping with a 3-value element (typically boron). In this case, the piezoresistive coefficient for the transversal and longitudinal loads are nearly the same with different signs. Therefore, a bridge can be constructed by mounting two resistors parallel and two restistors perpendicular to the applied stress or strain. This allows a high degree of miniaturization.

The silicon strain gauge (Si-DMS) have some advantages over the metal strain gauges but also some disadvantages. The main advantages of metal strain gauges are mechanical handling and low temperature coefficient of reactivity due to the good thermal adaptation to the DUT.

One advantage of the monocrystalline strain gauge is a result of the high gauge factor. The silicon gauge factor is about 80 whereas the metal strain gauge is approx. 2-3. A further advantage is the miniaturization, an area of $100 \mu \mathrm{m} .100 \mu \mathrm{m}$ is sufficient for a full Wheatstone bridge. High demands are placed on the overall geometry and packaging to take advantage of these benefits. 


\section{Structure of silicon strain gauge}

Silicon strain gauge (Si-DMS) are based on the n-doped (phosphorous) silicon wafer, $<100>$ orientation, with a resistivity of $R=3 \ldots$ $5 \Omega \mathrm{cm}$. With the help of MEMS technologies, the strain gauge can be produced. In Fig. 1, the layout is shown. The implantation parameters are configured for a sheet resistance of the piezoresistive resistance of about $200 \Omega / \square$ with a temperature coefficient of about $0.8 \% / 10 \mathrm{~K}$. The piezoresistive resistors are connected by low-implanted leads. Around the diffused resistors and leads there is an $\mathrm{n++}$ - doped guard ring. This serves to suppress parasitic current paths. The piezoresistive coefficient for the longitudinal and transverse effect are similar in magnitude

$\pi_{\mathrm{L}(100)}=71.810^{-11} \mathrm{~Pa}^{-1}$,

$\pi_{\mathrm{T}(100)}=-66.310^{-11} \mathrm{~Pa}^{-1}$,

but have opposite signs, thereby it is possible to build a concentrated full-bridge on a chip. It is also possible to build a spread full bridge with individual resistors. The required opposite change in resistance is achieved by the position of the Si-DMS on the spring body.

The footprint of the Si-DMS is $1000 \mu \mathrm{m} \cdot 500$ $\mu \mathrm{m}$. For the different applications, the sensors can be thinned down to $5 \mu \mathrm{m}$.

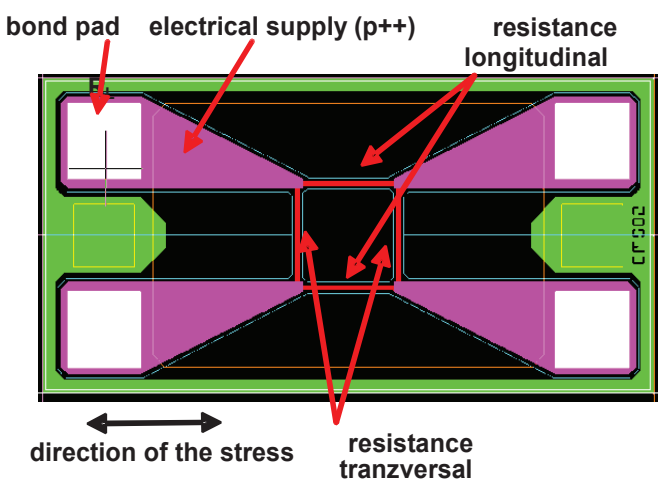

Fig. 1: Design of the silicon -strain gauge (Si-DMS)

The measured parameters from a selection of manufactured sensors are shown in Fig. 2. Of particular importance is a small detuning of the measuring bridge $\left(\mathrm{U}_{\text {offset } 30 \mathrm{C}}\right)$. In contrast to conventional strain gauge the bridge cannot subsequently be adjusted. Fig. $2 \mathrm{~b}$ shows the parameters of the individual resistances, they vary within a narrow range. The temperature coefficient of the individual resistors is mainly determined by the parameters of implantation and subsequent thermal treatment. In contrast, the temperature coefficient of the measuring bridge is mainly determined by the setup and the difference of the individual resistors.
Precision manufacturing for the system is available.

As previously reported, a temperature change always takes an influence on the primary measurement signal. In addition, the sensitivity is also temperature-dependent, in Fig. 3 the graph is shown. What is important is the reproducibility and the possibility for compensation.

\begin{tabular}{|c|c|c|c|c|c|}
\hline & $\begin{array}{c}R_{\text {bridge } 30 c} \\
\text { (in } \Omega, \text { bei } \\
150 \mu A \text { ) }\end{array}$ & $\begin{array}{c}U_{\text {offset } 30 \mathrm{C}}^{\text {(in }} \\
\mathrm{mV} \text { ) }\end{array}$ & $\begin{array}{c}R_{\text {bridgee } 800} \\
\text { (in } \Omega, \text { bei } \\
150 \mu \mathrm{A} \text { ) }\end{array}$ & $\begin{array}{l}\mathrm{U}_{\text {offset }} \\
80{ }^{\circ} \text { (in } \\
\mathrm{mV} \text { ) }\end{array}$ & $\begin{array}{c}\text { Temp. } \\
\text { coefficient } \\
\text { (in } \% / 10 \mathrm{~K} \text { ) }\end{array}$ \\
\hline Min & 4887.94 & -5.03 & 5085.77 & -5.25 & 0.7 \\
\hline Average & 4983.69 & -1.66 & 5183.41 & -2.07 & 0.73 \\
\hline Max & 5052.72 & 1.72 & 5253.66 & 1.02 & 0.74 \\
\hline Median & 4980.03 & -1.72 & 5179.09 & $-2,05$ & 0.73 \\
\hline $\begin{array}{l}\text { St. } \\
\text { deviation. }\end{array}$ & 42.90 & 1.76 & 44.08 & 1.66 & 0.01 \\
\hline CpK & 7.64 & 1.58 & 8.95 & 1.60 & 4.41 \\
\hline
\end{tabular}

Fig. 2: Electrical measurements on single chip, base are 1000 pieces

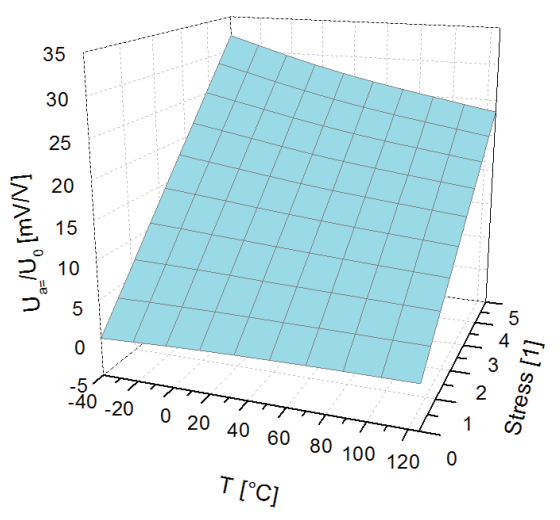

Fig. 3: Temperature coefficient of sensitivity

When using the silicon strain gauge attention has to be paid to the specific characteristics, in particular

- high young's modulus in comparison to the carrier foil of the metal strain gauge,

- no possibility of material adjustment to minimize thermo mechanical stresses

- low coefficient of thermal expansion (CTE),

- very small footprint of sensitive element.

From these properties, some of the basic requirements of the structure can be derived. The structure consists essentially a silicon strain gauge, the spring body and the bonding material, an adhesive or solder. Especially important is the mix of materials. 


\section{Assembling of chip and spring body}

In order to perform the function it is necessary that the strain gauge is perfectly connected by adhesive bond with the spring element. In this case, only the elongation of the spring body can be transferred without loss to the sensor. Due to the high Young's modulus of the silicon strain gauge, a material with equally high young's modulus is advantageous for joining. Good mechanical properties can be achieved by using a metal or glass solder. Depending on the material of the spring body, it produces high thermo mechanical stresses at a necessary processing temperature of about $450{ }^{\circ} \mathrm{C}$. Therefore, only a material with a small CTE is suitable for spring body, for example, silicon or kovar. Fig. 4 gives an overview of the expected thermo mechanical stresses for different material combinations. Alternatively, an adhesive may be employed for less good mechanical properties. Regardless of material, a thin layer of thickness from both the bonding material and the strain gauge is advantageous. $10 \mu \mathrm{m}$ for the silicon strain gauge and $5 \mu \mathrm{m}$ joining material have shown to give good result.

\section{Advantageous mechanical design}

The sensitive surface of the strain gauge is only $200 \mu \mathrm{m} \cdot 200 \mu \mathrm{m}$, wherein only a very small surface for the transmission of strain to the sensor is needed. This allows a design of the spring body which is for the same sensitivity significantly shorter and therefore stiffer. Fig. 5 shows the transmission of the strain from the spring body to the sensor. An epoxy-based adhesive has been used as a bonding material for simulation.

Fig. 6 shows a simple spring body with extensive regions of increased elongation. On the other hand, Fig. 7 shows a spring body optimized for the silicon strain gauge with concentrated areas of increased elongation. The advantage is the reduced flexibility of this spring body and higher sensitivity. The compliance of a perfect spring body is zero. The resilience of the spring body shown in Fig. 7 is only half as large as the conventional cantilever.

The results of the simulations are summarized in the charts of Fig. 8 and Fig. 9. A thin chip and $a$ thin layer of bonding material are advantageous. A low Young's modulus of the bonding material has to be compensated by a very thin layer thickness. The early forecasts have been confirmed.

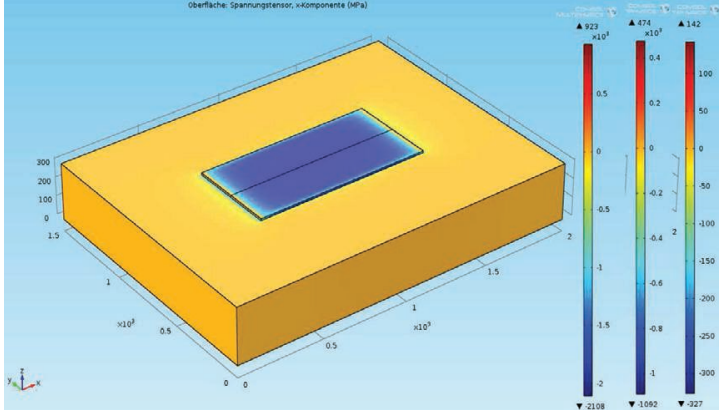

Fig. 4 Thermally induced mechanical stress of the silicon strain gauge due to the bonding temperature $\left(450^{\circ} \mathrm{C}\right)$ for different materials of the spring body, a) aluminum, b) steel, c) kovar

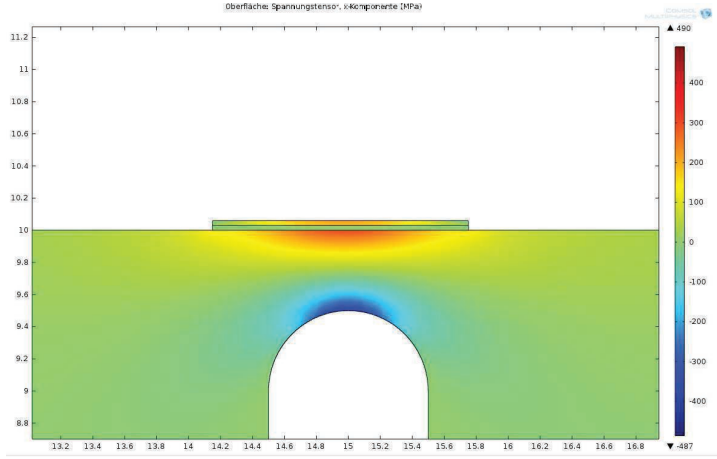

Fig. 5: Design of the silicon strain gauge, $1 \mathrm{~mm} \cdot 0.5$ $\mathrm{mm} \cdot 0.01 \mathrm{~mm}$, transfer of strain from the spring body on the sensor

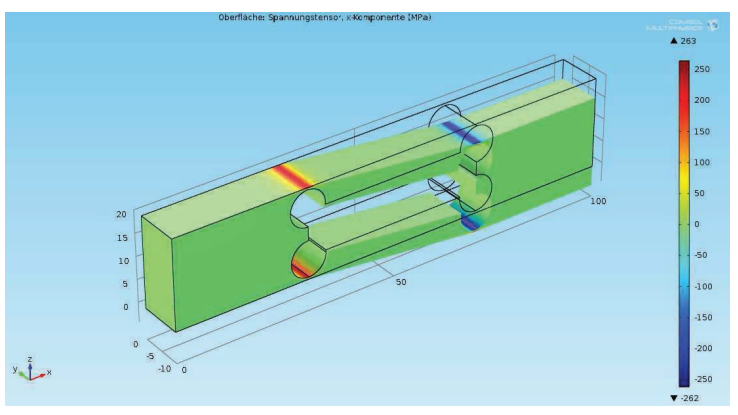

Fig. 6: Cantilevers for conventional metal strain gauge with compliant mechanisms

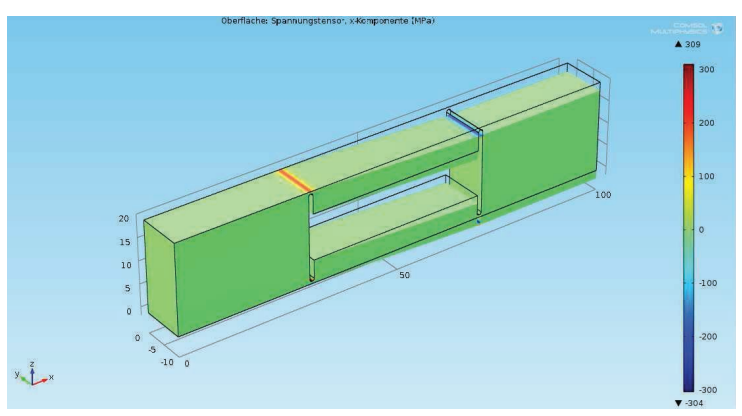

Fig. 7: Cantilevers for silicon strain gauge extremely concentrated compliance 


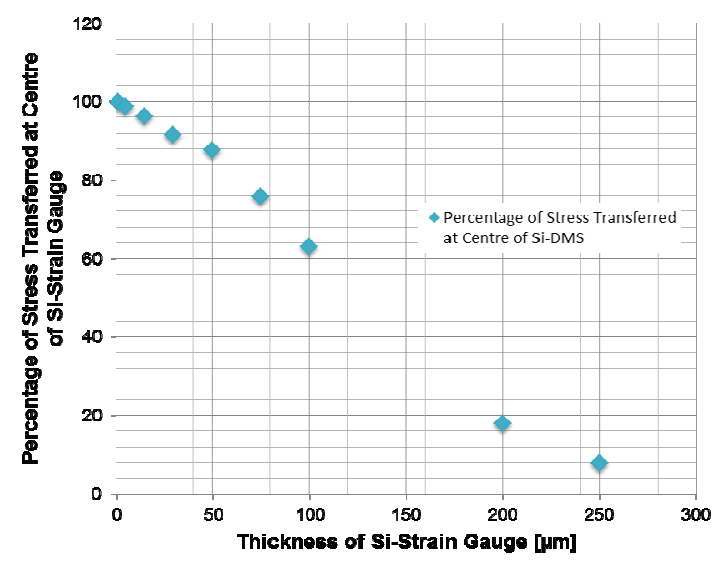

Fig. 8: Influence of Si-strain gauge thickness on sensor performance simulated

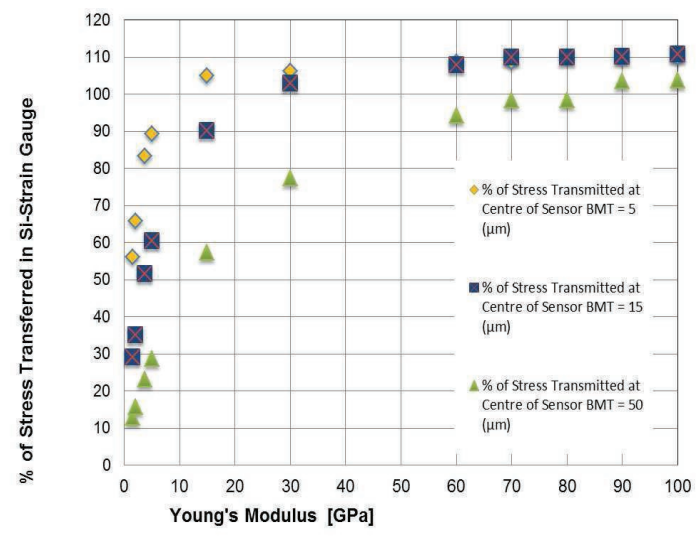

Fig. 9: Influence of Young's Modulus of bonded material \& bonded material thickness (BMT) on sensor performance

\section{Evaluation of the Silicon-strain gauge with various applications}

\section{Silicon cantilevers}

A good combination of materials is a silicon cantilever, a glass solder and the silicon strain gauge. There is no thermo-mechanical stress due to the identical CTE. On a macroscopic silicon cantilever, $3 \mathrm{~mm} \cdot 10 \mathrm{~mm} \cdot 30 \mathrm{~mm}$, the silicon strain gauge was evaluated. The cantilever is loaded in bending kind. The strain gauge is located at the position of maximum tension. In this article, the sensitivity is described as a function of the strain gauge. To determine the sensitivity as a function of the applied force, the geometry of the cantilever has to be taken into account. The sensitivity is related to the maximum normal stress in the outer fiber, which is determined by:

$$
\sigma_{\max }=\frac{M_{b}}{W_{a x}}=M_{b} \cdot \frac{a_{\max }}{l_{a x}}
$$

With:

$\sigma_{\text {max }}$ : Maximum normal stress

$M_{b}$ : Bending moment about the reference axis

$I_{a x}$ : Axial area moment of inertia

$a_{\text {max }}:$ Outer fiber distance

This stress is transmitted to the sensor and causes the required elongation to produce the measurement signal. For the presented strain gauge a sensitivity of

was determined.

$$
\mathrm{S}=0.4 \frac{m V}{\mathrm{~V} \cdot \mathrm{MPa}}
$$

\section{Aluminum cantilever}

A similar experiment was performed with aluminum cantilever. Due to the large difference in the CTE the strain gauge was glued with an adhesive. Fig. 6 shows a similar spring bar. This structure was chosen to compare silicon and metal strain gauge. Fig. 11 shows the positions of the sensors, which are positioned in the region of maximum stress, the compliant mechanisms. The sensors (1) and (2) correspond to Fig. 1. The bridge (3) consists of individual resistors, only uses the longitudinal effect. For this design, both the temperature dependence of the resistance and the thermo-mechanical stresses between aluminum and silicon must be taken into consideration. With the concentrated bridge (Fig. 1), these stresses cannot be separated from the measured value.

Compensation can only be electronic. If the individual resistances are connected to a measuring bridge (3), the thermo mechanical stresses at identical temperature can be compensated. A pair of resistors experiences compressive stress, the other pair tensile stress. The thermo-mechanical stresses are equal in both pairs and their effect on the signal bridge is negligible.

\section{Temperature dependence}

As explained previously, the temperature dependence of a silicon cantilever is solely based on the change in the resistors themselves. In an aluminum cantilever, the induced stressed due to difference in thermal expansion as well as elasticity of the materials (spring body, resistors and bonding) has to be taken into account. This leads to a stronger change in output voltage, as can be seen in Fig. 10. 


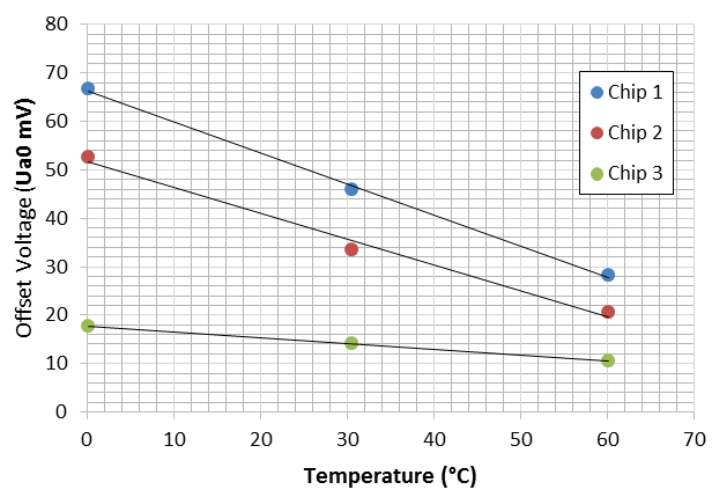

Fig. 10: Temperature dependence of zero point for Si-DMS on Al-cantilever

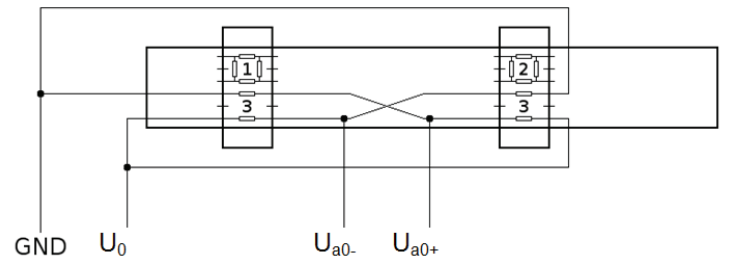

Fig. 11: bridges mounted on the spring body

Herein Chip 1 is a full bridge in the tensile stress region of the spring body, chip 2 is a full bridge in the compressive stress region of the spring body and chip 3 is a bridge consisting of 2 resistors in each region, as can be seen in Fig. 11).

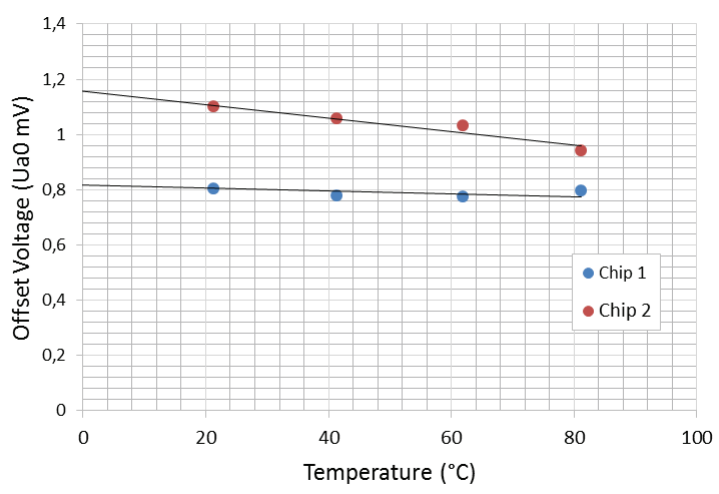

Fig. 12: Temperature effect of zero point on SiDMS mounted on silicon cantilever

When comparing the temperature dependence between a Si-DMS on an aluminum spring body (Fig. 10) with a Si-DMS on a silicon spring body (Fig. 12), it can be seen that the latter has a 100 times lower temperature dependence.

\section{Sensitivity}

The force sensitivity was measured using the OIML R 60 standard. It it we see chip 1 (blue and green), chip 2 (teal and yellow ) and chip 3 (crimson and orange). It can be seen that while the different sensors differ in the offset voltage, their sensitivities are almost identical with opposing signs. out of the tested sensors, the sensitivity only varies about $1.13 \% \mathrm{Sn}$ between all the sensors, while the offset voltage varies about 3.3\% Sn between the full bridges. The offset voltage of four resistors (chip 3) is relatively small. This stems from the fact, that any influences which cause the stress in 1 region of the spring body to decrease, cause an equal and opposite increase in the other section, factoring each other out.

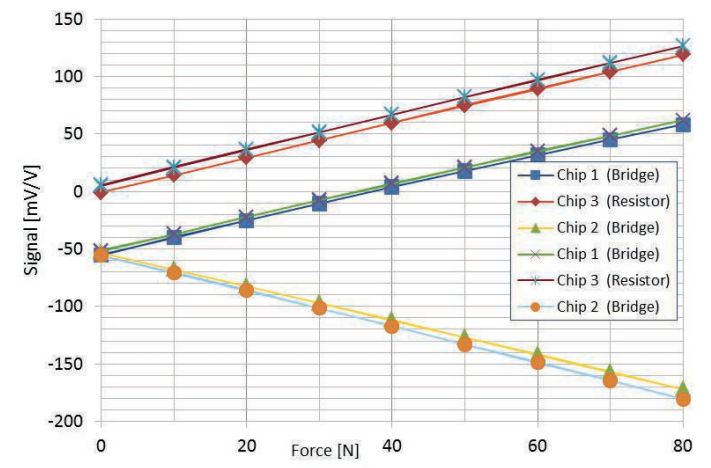

Fig. 13: Force-signal graph of the cantilevers

\section{Summary}

Silicon strain gauge sensors are highly applicable due to their high sensitivity (up to 50 times that of metal strain gauges) and their small size. The most preferable installation consists of two resistors in each region of the spring body. Due to the counteractive nature of this configuration, the influences of negative effects such as adhesive induced stresses are minimized resulting in reduced parasitic effects such as offset voltage or temperature dependence.

\section{Acknowledgment}

We thank the Bundesministerium für Wirtschaft und Energie (BMWi) the support for this project, Si-DMS (MF120100) financially.

\section{References}

[1] Mäuselein, Sascha, Oliver Mack, and Roman Schwartz. "Investigations into the use of singlecrystalline silicon as mechanical spring in load cells." Measurement 42.6 (2009): 871-877

[2] Rausch, Jacqueline Franziska Katharina. "Entwicklung und Anwendung miniaturisierter piezoresistiver Dehnungsmesselemente." (2012). Rausch, J. F. K. (2012).

[3] IPC/JEDEC-9704A, Printed Circuit Assembly Strain Gage Test Guideline, http://www.ipc.org/ feature 